\title{
Wireless instrumentation system experiment
}

\author{
Bibin Varghese $^{1, *}$, S. Sreelal ${ }^{1}$, S. Sreekumar ${ }^{1}$, P. Vinod ${ }^{1}$, M. N. Namboothiripad ${ }^{1}$, \\ Joseph $\mathrm{Lal}^{2}$ and $\mathrm{K}$. Anand ${ }^{2}$ \\ ${ }^{1}$ Avionics Entity, and \\ ${ }^{2}$ Advanced Space Transportation Systems, Vikram Sarabhai Space Centre, Indian Space Research Organisation, \\ Thiruvananthapuram 695 022, India
}

This article describes the design details of a Wireless Instrumentation System that was experimented in ISRO's recent Human in Space Project (HSP) Pad Abort Test (PAT) mission in piggy-back mode. The system consists of a few Wireless Sensor Nodes (WSNs) that acquire parameter data and a Wireless Base Station that collects these over an IEEE 802.15.4 compatible single-hop RF link and forward it to the telemetry subsystem. The circuit configuration, communication link and protocol as well as the measurement plan as adopted for HSP-PAT flight test are described to bring out the scalability of the architecture. The performance of the system in HSP-PAT mission is discussed in detail by way of PFA analysis results. All the parameters monitored through the system, including inertial ones such as acceleration and rotation were compared with reference data obtained from functional Telemetry Telecommand and Power and Navigation Guidance and Control chains and showed normal signatures. Maintaining an uninterrupted wireless communication channel in a hostile and crowded chamber like the Crew Module for all the nodes has called for a robust Medium Access Control (MAC) layer based on the industry-popular IEEE802.15.4 RF PHY. The requirement of such a robust MAC layer is established when the radio frequency links outage encountered in one of the WSN links during the interval of high vehicle dynamics is seen to be taken care of by the diverse link of the same node. A future roadmap towards self-powered wireless sensors is also outlined.

Keywords: Data acquisition, human in space project, launch vehicle telemetry, wireless sensor network.

\section{Introduction}

THE telemetry system that is used conventionally in Indian Space Research Organisation's launch vehicle follows a three-tier topology. This system, as described in our previous publications ${ }^{1,2}$, consists of sensors followed by signal conditioning Data Acquisition Units (DAUs), Data Processing Units (DPUs) and an Integrated telemetry Control Unit (ICU). The intra-stage as well as inter-

\footnotetext{
*For correspondence. (e-mail: bibin varghese@vssc.gov.in)
}

stage data communication among the units is carried out by party-line or dedicated serial buses in either half or full duplex configuration.

The above system architecture, though a modular and scalable one, has the drawback that it involves significant complexity in integration, especially with regard to the harnessing effort required at subassembly level. The installation and possible change of configuration require significant effort in documentation, inspection and testing. This problem is especially aggravated at stage-level subassembly of big launch vehicles like GSLV-MKIII intended for heavy lift capabilities. The related wiring, connectors, brackets and other mechanical parts to integrate the harness sum up to a mass of hundreds of kilograms. Further, the system does not render itself amenable to programming at subassembly or integrated vehicle level due to the wired interfaces that are required with the checkout computer at ground.

The harnessing complexity is mainly concentrated at the sensor output level. With sensors distributed over all the areas, including the outer structural periphery in a typical stage, this leads to a maze of wires running from multitude of locations to the DAUs located in an avionics deck mostly at the base or the inter-stage region. Wireless acquisition from sensors allows more flexibility and saving of time in integration, inspection as well as reduction in weight and volume. The ability of the wireless network to easily and quickly change its topology and augment an already installed network with in situ configuration or calibration of the sensors are new capabilities that bring more simplicity and flexibility in the system design. A Wireless Instrumentation System (WIS) was thus thought of as a solution where the distributed sensors communicate to a central unit through radio frequency (RF) link. The system thus consists of spatially distributed Wireless Sensor Nodes (WSNs) and a Wireless Base Station (WBS) which acts as a gateway from RF to main telemetry subsystem through intra-stage serial link. Some of the major considerations in the system design such as design of protocol and powering considerations are mentioned in some of our previous publications ${ }^{3,4}$. The major challenges in the system realization are design of the system in tune with advanced techniques such as those employed for Internet-of-Things (IoT), time synchronization between the nodes, immunity to external interferences, low 
volume, weight and power, and not compromising on reliability and robustness aspects that are so vital in the context of aerospace applications.

The piggy-back flight test of the system in Human in Space Project (HSP)-Pad Abort Test (PAT) mission served the primary objective of assessing the performance of a programmable, scalable, smart wireless instrumentation network within a challenging multi-path fading environment like the Crew Module (CM). Some of the other features tested newly are: (i) custom beaconing Medium Access Control (MAC) protocol of low latency compatible to IEEE802.15.4 (ZigBee) PHYsical layer (PHY); (ii) three types of diversities (spatial, frequency, temporal) to combat channel impairments in an enclosed environment; (iii) MEMS accelerometer and angular sensors with digital output to miniaturize the sensor nodes; (iv) remote programming of sensor nodes and packet telemetry; (v) reprogrammable flash-based Field Programmable Gate Array (FPGA) (ProAsic3), ultra low power microcontroller (MSP430) and ultra small (0402 size) passive components and (vi) Mixed Analog, Digital and RF printed circuit board (PCB) with low-cost FR4 material.

\section{Selection of a specific wireless technology}

There are broadly two types of wireless applications: point-to-point and point-to-multipoint. Applications falling under the first type are usually in the outdoor environment and require higher data rate and range. However, the applications under the second type usually operate in an indoor environment and have less data rate and range requirements. Intra-stage wireless data acquisition from sensors falls under the second category, and the system needs to work in a hostile closed metallic environment where the RF propagation conditions are more severe than a normal indoor case. As the volume of the chamber is small, round-trip delay of the RF signals will be less and the multi-path effects will persist for more duration within the chamber causing a higher root mean square (RMS) delay spread value.

Ultra-wide band (UWB) and infrared-based wireless sensor systems are experimented in the vehicle equipment bay of ARIANE 5 (ref. 5). Even though the UWB radio system is more tolerant to multi-path channel impairments, its use is limited due to the following reasons - its regulation is still not clear in many countries, possible interferences from vehicle S-band and C-band systems, availability of very few vendors providing IEEE802.15.4.aUWB compliant COTS radio. Infrared systems require extra set-ups like reflectors to make the non-line-of-sight (NLOS) communication possible. Temporal and spatial diversities have been used to improve the reliability of wireless communication ${ }^{6}$. However, the number of listeners required to provide spatial diversity made the scheme less attractive. Also, the re-transmissions in many slots with a different pseudo noise (PN) sequence to provide temporal diversity made the sensor node hardware more complex and less interoperable.

Spatially distributed redundant Access Points (APs) are used to reliably communicate without sacrificing end-toend latency even in NLOS conditions ${ }^{7}$. The synchronization between the APs and the requirement of transmitting the same message from all the APs simultaneously makes this scheme less attractive.

Other than the communication robustness requirement, we have also considered the following important points in selecting a specific wireless technology.

(1) Amenability for low power operation.

(2) Availability of standard and commercial off-theshelf (COTs) components.

(3) Availability of a wider unlicensed band for scalability as well as diversity of operation.

(4) Operation at a higher frequency so that the electromagnetic radiation effect on nearby circuits and devices will be less due to higher attenuation. This also enables the use of a compact RF module.

IEEE802.15.4 standard for Low Power Wireless Personnel Area Networks (LR-WPAN) meets the above requirements. It is used in the major standards for industrial automation like Wireless HART (ref. 8), ISA 100.11a (ref. 9) and WIA-PA (ref. 10). It is also recommended by Consultative Committee for Space Data Systems (CCSDS) for short-range launch vehicle applications.

\section{IEEE802.15.4 PHY}

Figure 1 shows PHY of the IEEE802.15.4. It uses an Offset Staggered Quadrature Phase Shift Keying (OQPSK) with half sine pulse shaping at the modulator side which is equivalent to the Minimum Shift Keying (MSK) and allows usage of efficient power amplifiers. The Direct Sequence Spread Spectrum (DSSS) technique will enable the system to tolerate multi-path fading. Spreading by a factor of eight gives a jamming margin of $9 \mathrm{~dB}$ from other co-channel RF transmissions like Bluetooth and WiFi. The penalty of requiring a higher bandwidth due to spreading is justified due to the availability of larger bandwidth in the $2.4 \mathrm{GHz}$ ISM band. Availability $16 \mathrm{RF}$ channels spaced by $5 \mathrm{MHz}$ in this band permits multiple simultaneous RF links which can be used by multiple systems or by the same system for frequency diversity purpose. System data rate is $250 \mathrm{kbps}$ ( $220 \mathrm{kbps}$ useful data rate) and the air signalling rate is 2 Mchips/sec after spreading.

\section{Selection of MAC layer}

Industrial standards mentioned above have employed reliable communication measures at the MAC level. However, 


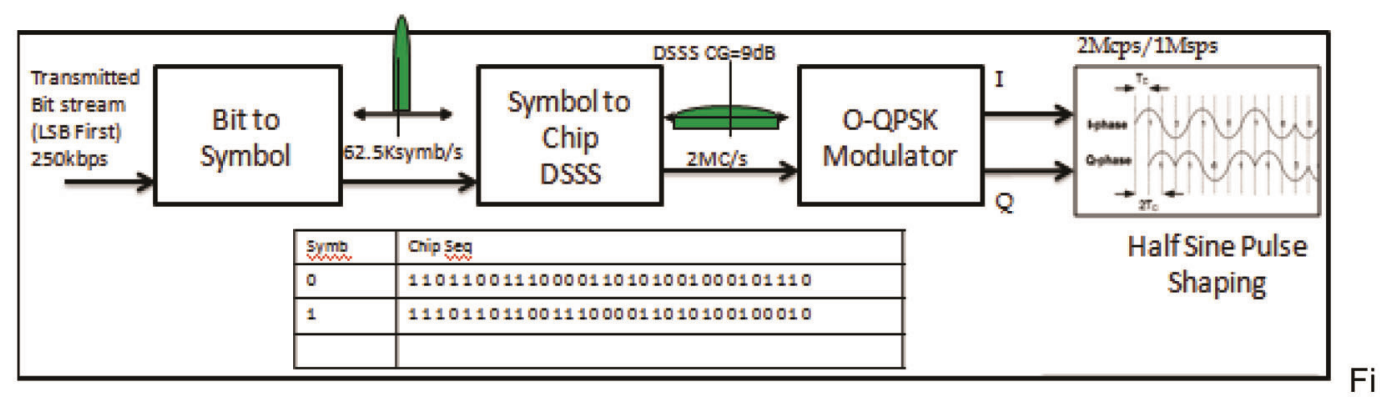

Figure 1. PHY of IEEE802.15.4.

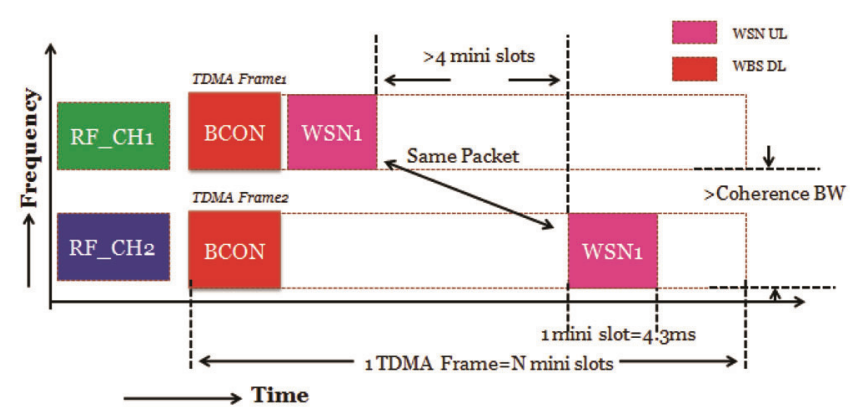

Figure 2. WIS MAC layer.

the simplicity and latency of the system is compromised in these standards and cannot be used in a launch vehicletype application where the mission duration is of few minutes and the latency of communication should be as low as possible. Desirable features for a robust and efficient MAC for aerospace applications are given below.

- Robust communication with all the nodes, irrespective of their location within the permitted operating range.

- Minimum latency for real-time data acquisition.

- Efficient bandwidth utilization independent of the data requirements of each node.

- Deterministic time slots depending on the data rate requirements of each node.

- Scalability and programmability.

- Simple hardware and software requirements.

\section{Error manifestations in an indoor aerospace environment}

The propagation environment, which mainly includes reflective surfaces and hindering objects causes multi-path reflection as well as shadowing of the signal waveform, and the distortion translates into incorrect DSSS chip sequence decoding via the OQPSK demodulator, ultimately resulting in bit errors. Signal spreads of the order of tens or hundreds of nanoseconds are typically observed in industrial environments ${ }^{11}$, with log-normally distributed large-scale fading and small-scale fading closely following a Rician distribution ${ }^{12}$. Despite Rician- and Rayleigh-like behaviour of fading, studies report fading dips of 30-40 dB (ref. 11). Since modulation and pulseshaping are strictly defined by the standard, any alterations in that sense would breach the compliance with the standard and disable interoperability. Another way of improving the communication robustness is to incorporate suitable techniques in the MAC layer. Issues with the conventional way of retransmission are that it is often not possible in the very next timeslot and will reduce the throughput of the system as well. Another way of handling this is to use two diverse paths for communication with minimal overhead at the WSN side. This can be achieved by allowing TX/RX diversity at the coordinator side and TX diversity at the sensor node side. Based on the above points a simple and efficient FDMA + TDMA MAC layer optimized for the IEEE802.15.4 PHY has been proposed in our previous work $^{3}$ (Figure 2). In this MAC, the coordinator, i.e. WBS will handle two RF networks in two diverse RF frequencies which are kept apart by more than the coherence bandwidth of $2.4 \mathrm{GHz} \mathrm{RF}$ band to exploit the frequency selectivity of the channel. Each RF channel is time-division multiplexed into a programmable number of mini slots. $N$ number of such mini slots will constitute a Time Division Multiple Axis (TDMA) frame. WBS will send broadcast beacons in the first mini slot (third micro slot) of every TDMA frame and the listening WSNs will adjust their internal timer to synchronize with the WBS timing. WSNs can send their data in the programmed time slots and a time drift of $\pm 22 \mu$ s between the WSNs is permitted in this design, which is long enough to be met by the Micro Control Unit (MCU). Same data will be sent in both the RF channels by the WSN and these slots are kept more than four mini slots apart, which gives temporal diversity and allows the system to tolerate short-term noises due to interferences in one or both of the RF channels. Frequency diversity will allow the system to tolerate a single link failure or occasional outages in the RF channels. Implementation of this system will call for a dual radio in WBS and a single radio in the WSN side. This allows low power, low weight and low volume implementation of the WSNs, which are required more in numbers. WBS which 
is more data-centric can be realized with powerful FPGAs or processors. WBS receiver antennas can be kept more than $\lambda / 4$ apart to achieve spatial diversity also, which again helps the system to further tolerate multipath fading related issues as the antennas will see independent fading and the probability of a deep fade in both the antennas simultaneously is very less.

\section{Wireless Instrumentation System}

WIS is designed as a distributed smart sensor network system in a single hop star network operating in the $2.4 \mathrm{GHz}$ ISM band complying with the PHY and MAC layers discussed in the previous section. WSNs with integrated sensors and external signal conditioning capability communicate to the WBS that acts as an RF to telemetry subsystem gateway by wireless means in a cell radius of $10 \mathrm{~m}$. The communication between WBS and WSNs is in terms of beacon and data packets which have the same format as that of IEEE802.15.4 PDU (ref. 13). Combining tri-axial accelerometer, tri-axial gyro angular sensor, temperature sensor, low and high level analog signal monitoring, embedded processing and wireless communications, the WSNs operate within a fast, synchronized, scalable network. The medium can be a moderate Rayleigh or Rician fading indoor environment due to the robust MAC layer.

\section{Wireless Base Station}

WBS is the heart of WIS. Figure 3 shows its hardware block diagram. Central to WBS is a re-programmable ProAsic3 flash-based reprogrammable FPGA (ProAsic3) which will control the different subunits in the card. There are two IEEE802.15.4 PHY-compliant radios (TImake CC25XX radio) controlled by the FPGA through two independent four-wire SPI interfaces operating at $4 \mathrm{MHz}$. PHY and Lower MAC (LMAC) layer are implemented in CC25XX. Upper MAC (UMAC) layer is implemented in the FPGA which will control channel access and packetization of data. Figure 4 shows functional block diagram of the FPGA logic. In order to boost the power of the radio output and the weak input signal received, a power amplifier-cum-LNA IC (TI-make CC25XX) is provided at the RF front end of both the radios. $\mathrm{RF}$ power can be varied from -8 to $+18 \mathrm{dBm}$ by programming the appropriate $\mathrm{CC} 25 \mathrm{XX}$ registers. Receiver sensitivity of $-97 \mathrm{dBm}$ ( $1 \%$ packet error rate (PER)) is obtained in the actual card. WSN data received in two RF links are put in internal first-in-first-out memory (FIFOs) made of FPGA static RAM blocks and sent in the same order with suitable header to the main telemetry through respective RS485 links. WBS programmable parameters are stored in a FLASH memory inside the FPGA and a JTAG interface is provided for in-circuit programming of
FPGA. Unlike conventional RF designs which use costly RT/duroid PCBs for the RF part, and digital and analog designs on separate PCBs, WBS has used a single low-cost and low-loss HiTg FR4 material for the entire circuit with proper layout techniques. The usage of reprogrammable ProAsic3 FPGA has reduced the design cost by reducing the number of external components (like RAM, FIFO, Flash, etc.) and has given more flexibility and ease in the logical design of the system. Ultra-small (0402 sized) passive components were used in the RF side, which not only improved the RF performance but also reduced the RF circuit footprint on the card.

\section{Wireless Sensor Node}

WSNs are a spatially distributed autonomous sensor-cumdata acquisition system which pass their data to the central WBS by wireless means in a time synchronous manner. Central to the WSN is an ultra-low power micro controller (MSP430 series MCU) operating at $16 \mathrm{MHz}$ clock derived internally. Figure 5 shows the hardware block diagram of WSN. Analog high-level channels (0-2.5 V, $488 \mathrm{sps}$ ) are provided using the internal Analog-to-Digital Converter (ADC) (12-bit SAR) of the MCU itself. They can also be configured for internal supply (AVDD/2) monitoring by writing the appropriate registers of the MCU. There are two programmable (gain = 1, 2, .., 128 and data rate $=31.5,61, \ldots, 488$ ) analog low-level channels provided by two sigma delta ADCs (ADS12XX) which are interfaced to MCU by Serial Peripheral Interface (SPI) means. Both of the low-level channels can be

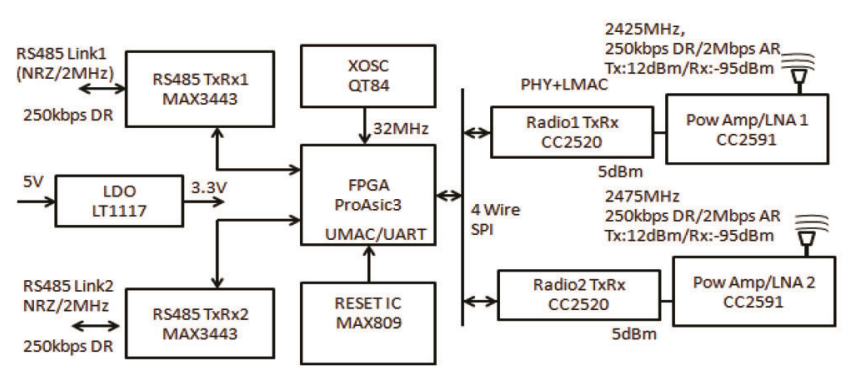

Figure 3. WBS hardware block diagram.

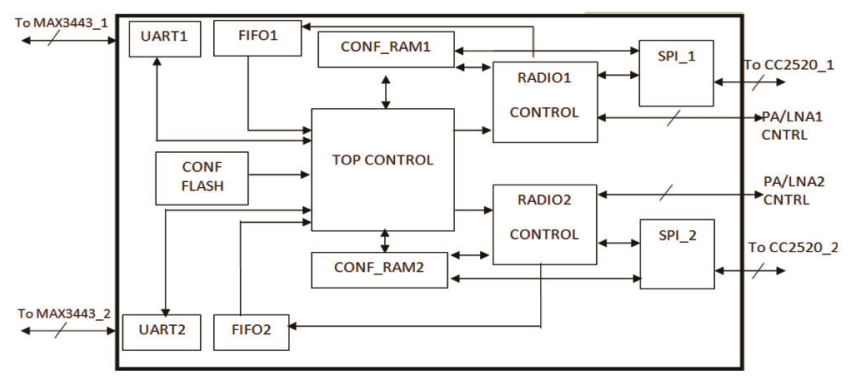

Figure 4. WBS FPGA logic functional block diagram. 
connected to a RTD/pressure/voltage channel with the provision of current excitation for RTD channels. ADXLXXX is a MEMS-based three-axes digital accelerometer sensor provided inside the WSN to measure accelerations up to $\pm 16 \mathrm{~g}$. With only $140 \mu \mathrm{A}$ current consumption and typical supply voltage of $3.3 \mathrm{~V}$, it reaches a resolution of $4 \mathrm{mg} / \mathrm{LSB}$ with 13 bits output data for each axis. Similarly, L3GDXXX is a three-axes gyro angular sensor to measure rotations up to $2000 \mathrm{dps}$ that consumes $5 \mathrm{~mA}$ with a supply voltage of $3.3 \mathrm{~V}$. It has a resolution of $5 \mathrm{mdps} /$ digit with 16 bits of output data for each axis. Both sensors are interfaced to the MCU by SPI means. A temperature sensor (TMP36) is also provided in the card to monitor the local temperature. CC25XX radio $+\mathrm{CC} 25 \mathrm{XX} \mathrm{PA} / \mathrm{LNA}$ combination forms the $\mathrm{RF}$ interface similar to that of WBS. Radio MAC as well as polling of the peripherals are operated by the MCU and are clocked from an $8 \mathrm{MHz}$ buffered clock sourced by $\mathrm{CC} 25 \mathrm{XX}$ radio to meet the MAC timing margin.

WSN MCU programming is done through JTAG interface provided in the board. WSN node parameters are programmed through the RF interface when WSN is switched on in the programming mode. The data rate of a single WSN is the sum of the data rates of all the selected channels, and the required data rate is set by appropriately formatting the TDMA frame. Maximum data rate for a single low level or high-level channel is limited to 488 sps and those of accelerometer and angular sensor channels are limited to $400 \mathrm{sps}$. Each WSN can be independently programmed to accept a maximum of eight channels (internal or external) from various internal and external sensors (16 nos), and it gives out packetized data

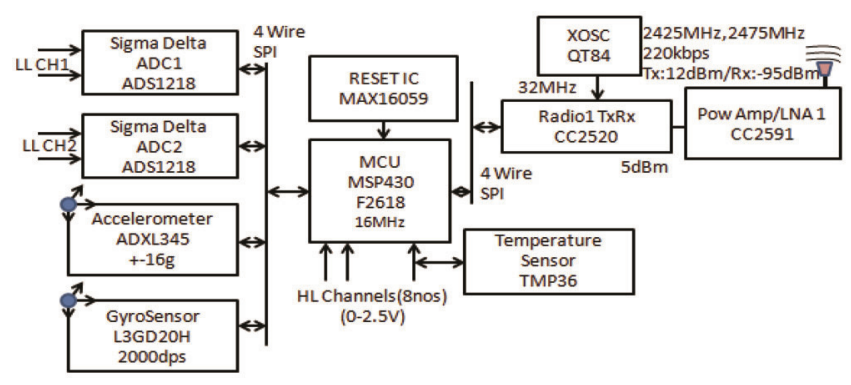

Figure 5. WSN hardware block diagram.
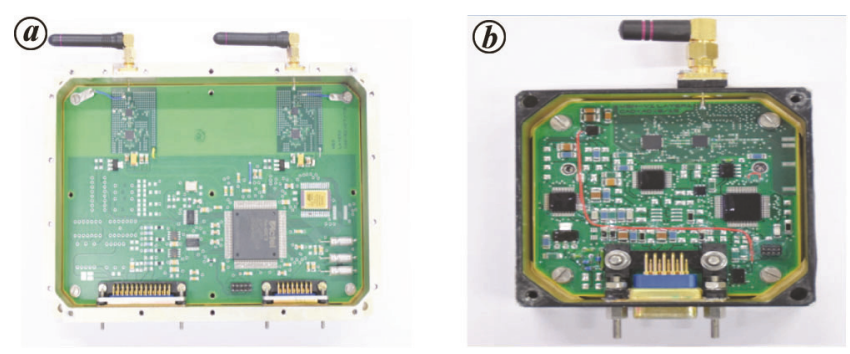

Figure 6. $\boldsymbol{a}$, Wireless Base Station. $\boldsymbol{b}$, Wireless Sensor Node. to the downstream WBS in two diverse RF channels. WSN design also used 0402-sized passives at the RF side and HiTg-based PCB for the circuit design. Figure $6 a$ and $b$ shows the WBS and WSN modules after wiring and mechanical assembly.

COTS components are used extensively for system implementation since they are essential for miniaturized, low-power, low-cost and software programmable hardware implementation. The system design principles, though, may be extended to mission critical aerospace applications with hardware based on military grade devices. Such an implementation will retain all the systemlevel advantages such as un-tethered, plug-and-play capability and integration harness simplicity as presented here.

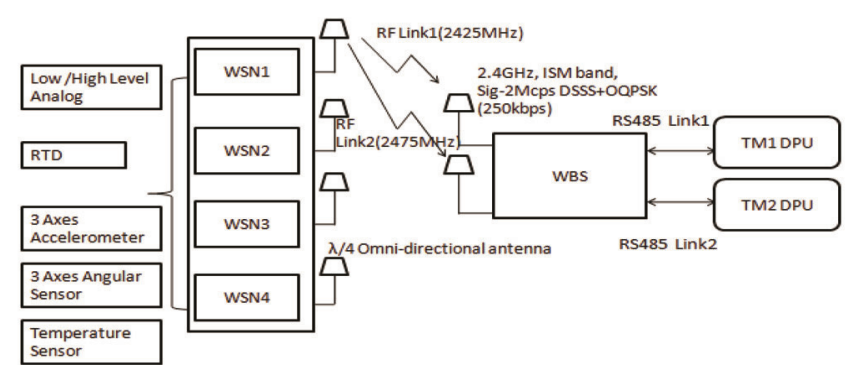

Figure 7. WIS test set-up in HSP-PAT.

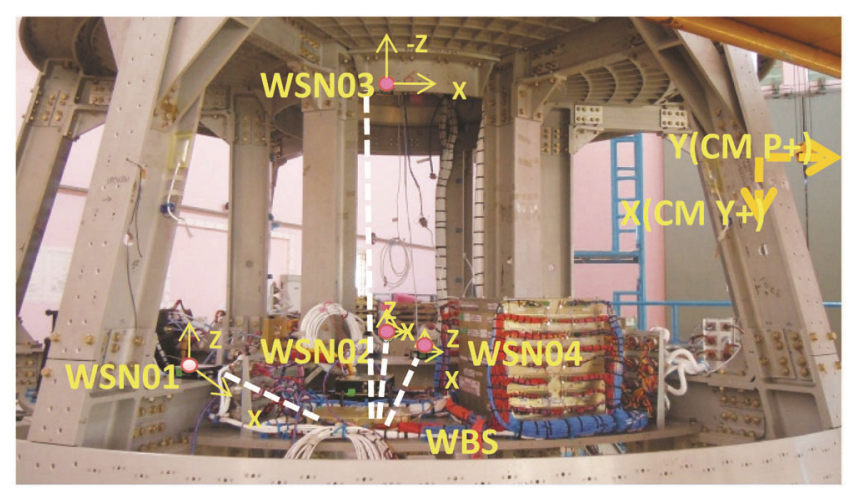

Figure 8. WSN locations inside the Crew Module.

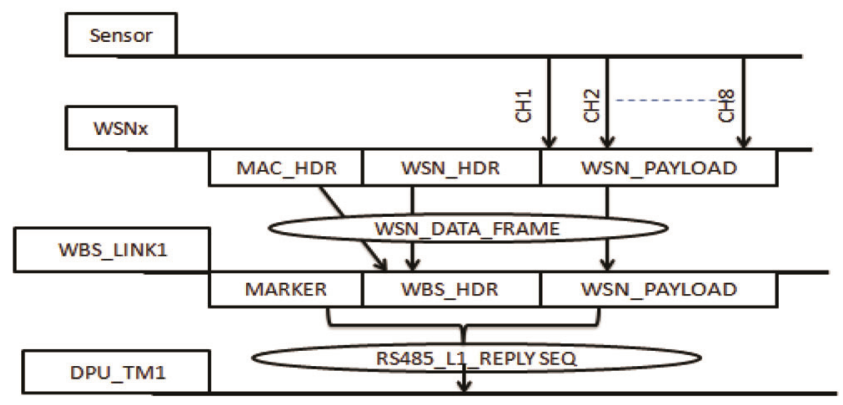

Figure 9. WIS data flow. 
Table 1. WSN sensor configuration

\begin{tabular}{lcccccc}
\hline Node & CH1 & CH2 & CH3 & CH4 & CH5 & CH6 \\
\hline WSN1 & ${ }^{1}$ VOL & ${ }^{2}$ RTD & RTD & ${ }^{3}$ ANG_X & ANG_Y & ${ }^{4}$ TEMP \\
& $0-2.5 \mathrm{~V}, 61 \mathrm{sps}$ & $70-200 \Omega, 61 \mathrm{sps}$ & $70-200 \Omega, 61 \mathrm{sps}$ & $\pm 245 \mathrm{dps}, 200 \mathrm{sps}$ & $\pm 245 \mathrm{dps}, 200 \mathrm{sps}$ & $0-70^{\circ}, 61 \mathrm{sps}$ \\
WSN2 & VOL & RTD & RTD & ${ }^{5}$ ACC_X & ACC_Y & TEMP \\
& $0-2.5 \mathrm{~V}, 61 \mathrm{sps}$ & $70-200 \Omega, 61 \mathrm{sps}$ & $70-200 \Omega, 61 \mathrm{sps}$ & $\pm 16 \mathrm{~g}, 200 \mathrm{sps}$ & $\pm 16 \mathrm{~g}, 200 \mathrm{sps}$ & $0-70^{\circ}, 61 \mathrm{sps}$ \\
WSN3 & VOL & ACC_Z & ANG_X & ANG_Y & ANG_Z & TEMP \\
& $0-2.5 \mathrm{~V}, 61 \mathrm{sps}$ & $\pm 16 \mathrm{~g}, 200 \mathrm{sps}$ & $\pm 245 \mathrm{dps}, 200 \mathrm{sps}$ & $\pm 245 \mathrm{dps}, 200 \mathrm{sps}$ & $\pm 245 \mathrm{dps}, 200 \mathrm{sps}$ & $0-70^{\circ}, 61 \mathrm{sps}$ \\
WSN4 & VOL & ANG_Z & ACC_X & ACC_Y & ACC_Z & TEMP \\
& $0-2.5 \mathrm{~V}, 61 \mathrm{sps}$ & $\pm 245 \mathrm{dps}, 200 \mathrm{sps}$ & $\pm 16 \mathrm{~g}, 200 \mathrm{sps}$ & $\pm 16 \mathrm{~g}, 200 \mathrm{sps}$ & $\pm 16 \mathrm{~g}, 200 \mathrm{sps}$ & $0-70^{\circ}, 61 \mathrm{sps}$ \\
\hline
\end{tabular}

${ }^{1}$ Internal supply voltage monitoring. ${ }^{2}$ Temperature measurement from external RTD sensor. ${ }^{3}$ Angular sensor $x$-axis monitoring. ${ }^{4}$ Internal temperature sensor monitoring. ${ }^{5}$ Accelerometer $X$-axis monitoring.
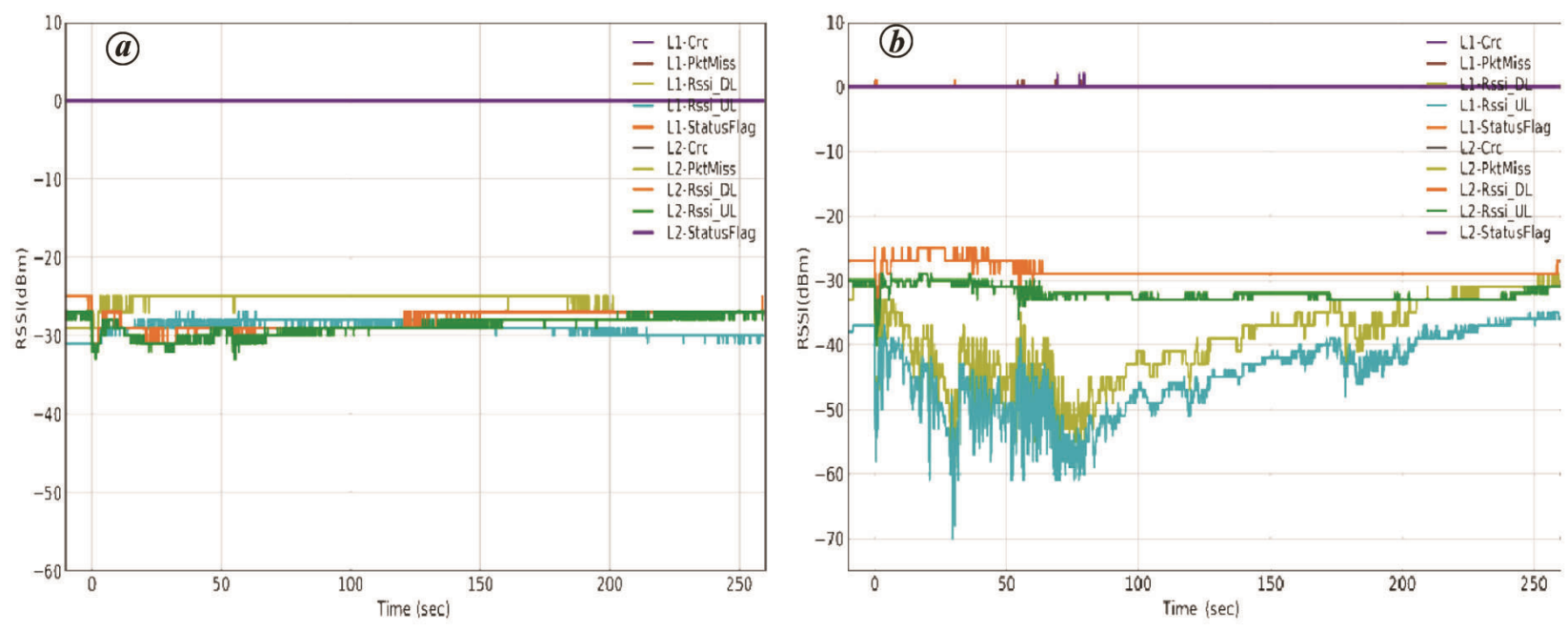

Figure 10. $\boldsymbol{a}$, WSN01 RF link parameters. $\boldsymbol{b}$, WSN03 RF link parameters.

\section{Experimental set-up in HSP-PAT mission}

WIS is flown in the HSP-PAT mission in piggy-back mode to evaluate the performance of the system in actual flight conditions. Figures 7 and 8 show the configuration of the actual experiment. Four WSNs were used within the $\mathrm{CM}$ at different locations (Figure 8), (farthest node is at a distance of about $2.2 \mathrm{~m}$ ). Table 1 gives the sensor configuration of each of the WSN. The WBS is interfaced to two Data Processing Units (DPUs) by means of wire line (serial RS485). This environment imposes two major design challenges: shadowing caused by large-sized objects between the WBS and sensor nodes, and significant multi-path propagation as $\mathrm{CM}$ is an enclosed metallic housing. Size of the TDMA frame is fixed as 21 mini slots to adapt to the lowest sampling requirement and the RF power of both WBS and WSN is programmed to be $12 \mathrm{dBm}$, which gives a design margin better than $35 \mathrm{~dB}$ in a $3 \mathrm{~m}$ radius. Both WBS and WSNs used mono pole $\lambda / 4$ whip antennas of minimum $-2 \mathrm{dBi}$ gain and have maximum gain in the $X-Y$ plane. Nodes are deployed in such a way that the relative antenna orientation is aligned optimally with the WBS antenna orientation with the adjust mechanism of the antennas used. Due to the presence of many metallic objects, a true line-of-sight communication from the WBS to sensor nodes was not feasible in CM.

Figure 9 shows the data flow from a single WIS RF link to telemetry TM1 link in HSP-PAT. WSN packets include the selected sensor data in the payload part and RF/MAC parameters like received signal strength (RSSI), Link Quality Indication (LQI), cyclic redundancy check (CRC) checksum, etc. in the header part. Header part of WSN data will be modified by WBS to append an error flag, which indicates different error conditions like slot mismatch, beacon miss, FIFO overflow, received signal strength, etc. FPGA logic within the WBS is able to measure the slot mismatch up to $\pm 860 \mu$ s with a resolution of $1 \mu \mathrm{s}$. Further this packet will be prefixed with a 3-byte marker before sending to the DPU over RS-485 links.

WSN will post the same data in the alternate WBS RF link also and will be routed to the TM2 link of telemetry by WBS. At ground the best packet is selected (selection combining) based on the CRC pass flag. The measurement plan of WSNs is such that each type of sensor is addressed twice over all the nodes and the total bandwidth 


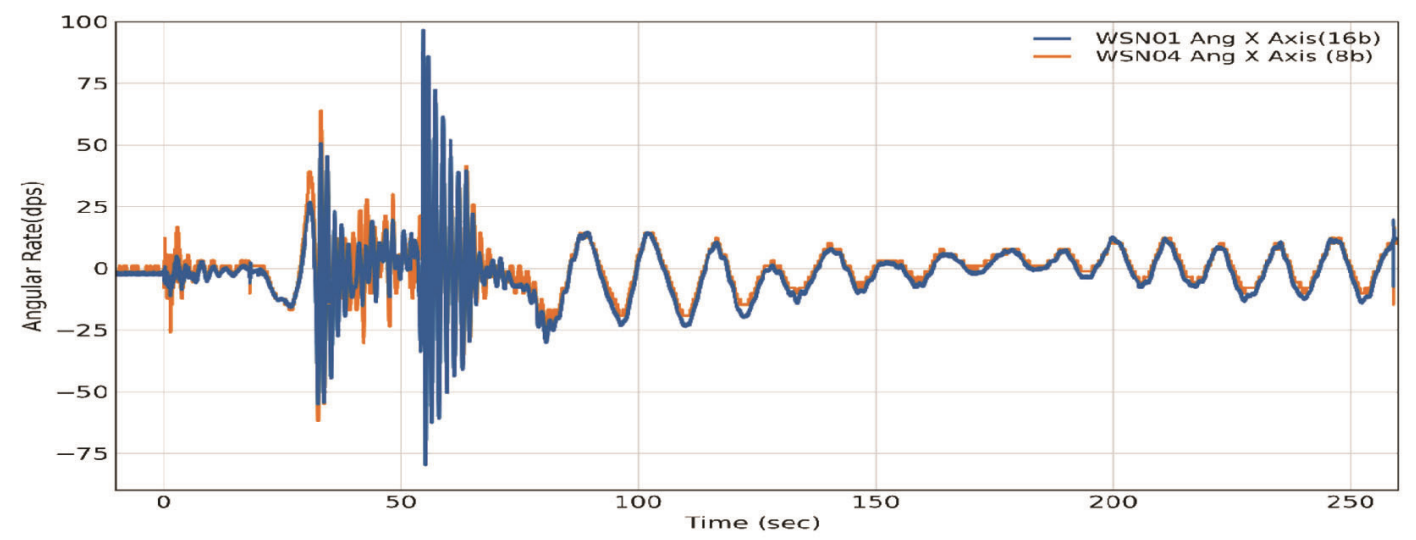

Figure 11. Angular sensor $x$-axis comparison.
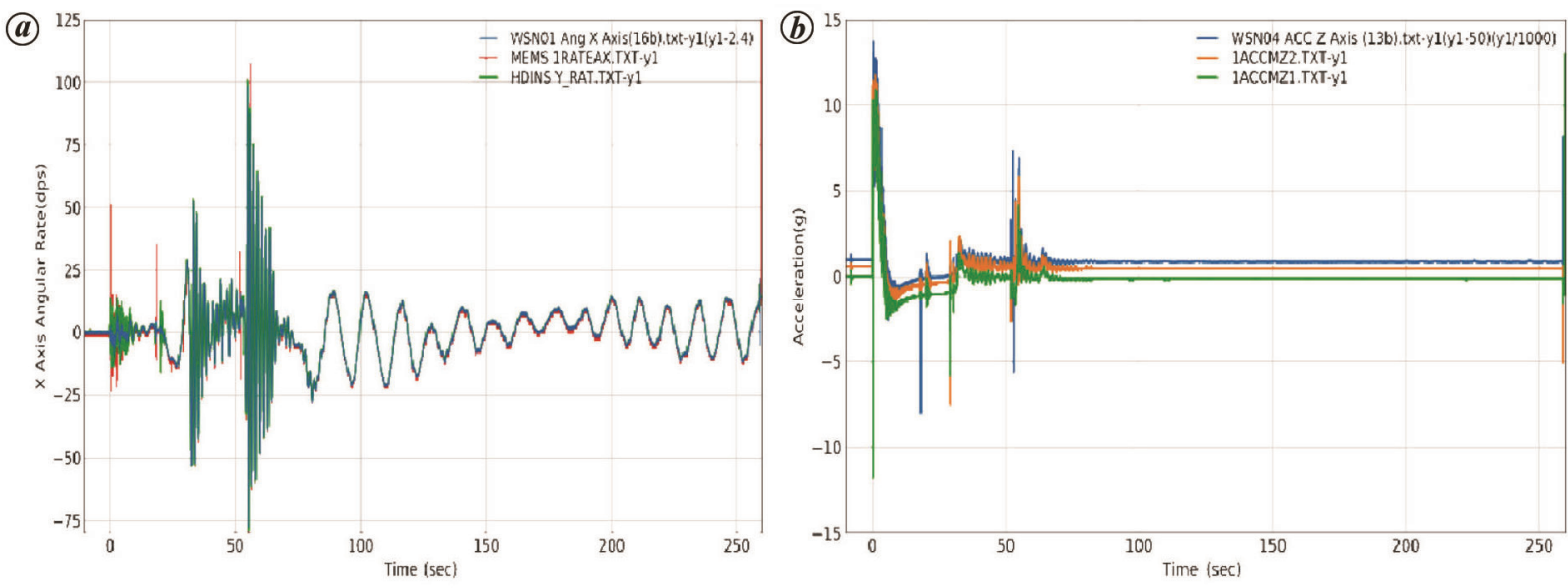

Figure 12. Comparison with reference sensors: $\boldsymbol{a}$, angular sensor ( $x$-axis); $\boldsymbol{b}$, accelerometer ( $z$-axis).

allocation is $32 \mathrm{kbps}$ in a single telemetry link. Reference sensors were identified for each type of measurement in the functional chain for data comparison.

\section{Performance in flight}

WIS performance is verified from the data obtained from the pre-flight tests and all the RF performance parameters are in the expected range. Analog channel and sensor parameters are also verified by comparing with reference values from the functional telemetry chain. WIS performance was normal in the actual HSP-PAT mission for the total mission duration of approximately $260 \mathrm{sec}$. All the 24 parameters measured by WIS were received through the vehicle telemetry without any error, and were compared and found to match closely with the reference parameters monitored separately in the functional chain.

Few packet misses were observed in WSN03 RF Link1 alone between $T=0$ and $T=100 \mathrm{sec}$. RF Link1 uplink and downlink RSSI values also show a dip (to $-70 \mathrm{~dB}$ ) during this time (Figure $10 a$ and $b$ ). The same data are received in RF Link2 without error. The role of RF hardware in WBS and WSN03 is ruled out as the same transmitter at WSN03 is used to transmit in the other RF channel, and the same receiver at WBS is used to receive data from other WSNs in the same frequency, and both these paths showed normal performance. No other errors like slot mismatch or FIFO overflow occurred during this time for WSN03. Hence the probable cause for RF signal dip is a frequency-selective fading that occurred in RF Link1 path from WSN03 to WBS during this time due to the relative movement of some loosely tied foam material between WBS and WSN03 during the period of high dynamics.

Analog parameters of same type monitored in multiple WSN packages are compared and show good match with each other (Figures 11 and 12). Slight difference in the accelerometer data as shown in Figure $12 b$, is probably due to the difference in mounting locations of these nodes. Angular parameters show good match with each other and also with the reference sensors in the functional chain (Figures 11 and $12 a$ ). Temperature and voltage 

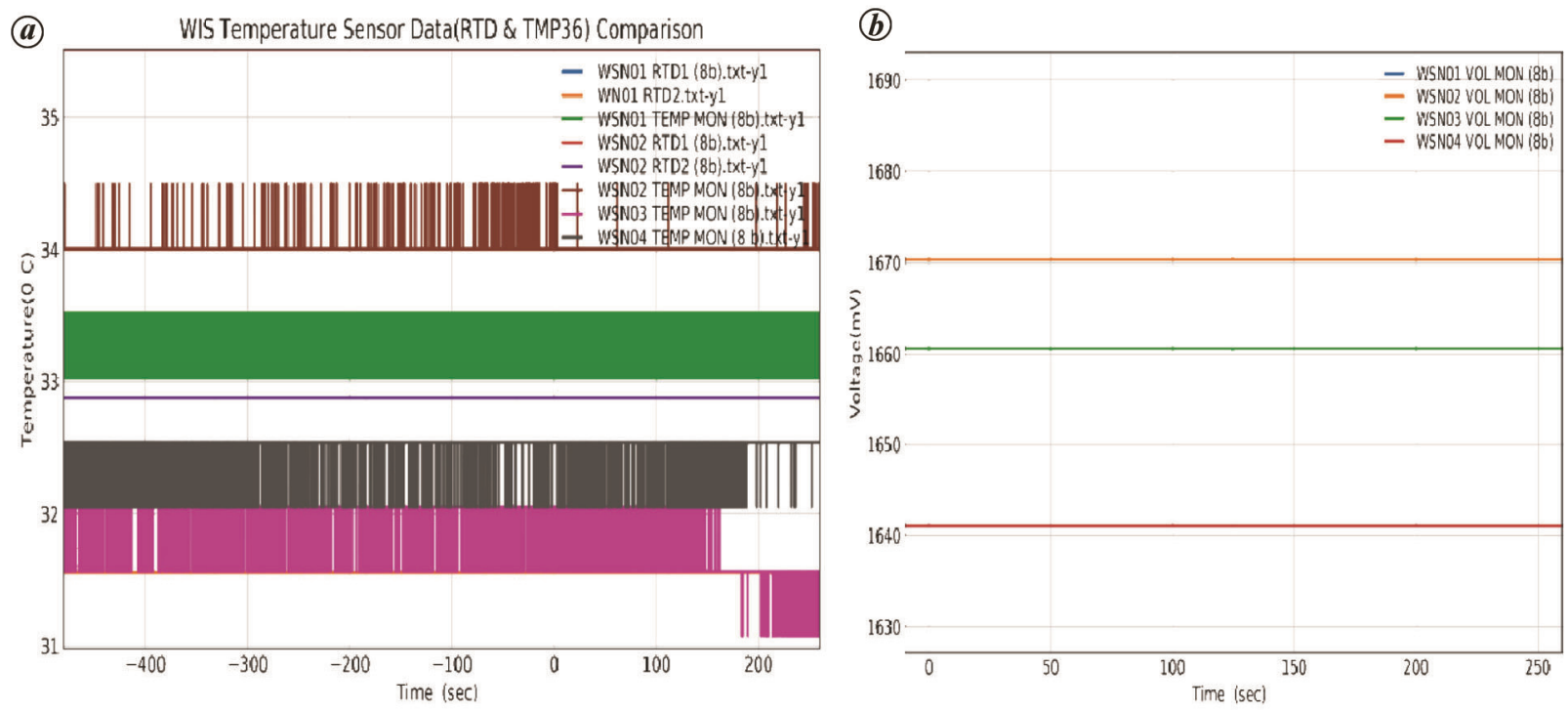

Figure 13. $\boldsymbol{a}$, Temperature monitoring. $\boldsymbol{b}$, Internal supply monitoring.

measurements also show good match with each other (Figure 13).

The mean received signal strength values measured at WBS from different WSNs varied between $-25 \mathrm{dBm}$ (for WSN01) and $-40 \mathrm{dBm}$ (for WSN04). This variation is as expected with respect to the distance from WBS and is well within the sensitivity limit $(-95 \mathrm{dBm})$ of WIS. The variation of signal strength for individual sensor nodes was minimal, except for the WSN03 RF Link1 as discussed earlier. The error flag which has captured the different error conditions of WSNs, including slot mismatch, beacon miss, FIFO overflow, etc. has shown a value of zero throughout the mission duration for all the WSNs, except the case of WSN03 RF Link1. Maximum time slip observed is only $\pm 6 \mu$ s during the temperature testing of WSNs at ground and the slip measured during the flight was only $\pm 3 \mu$ s.

The CM of HSP-PAT mission is a closed capsule with potential multi-path RF propagation through multiple reflecting surfaces. Therefore, operation of the system in an enclosed environment as is envisaged in any other aerospace application is tested. The system performance gives sufficient confidence for its application in such scenarios. The vulnerability of the system in an explosion environment is similar to that of any other avionics system and needs to be studied further. As part of system test and evaluation, the WBS and WSN subsystems have been subjected to vibration tests mounting them on a shaker table. The system response to shock stimulus is planned to be experimented and analysed as part of qualification tests for study on shock survivability.

\section{Conclusion}

This article presents a proof-of-concept for a IEEE802.15.4-based WSN for aerospace applications in an actual flight experiment. Based on the pre-flight and actual flight experimental results, we have demonstrated a WIS for the harsh aerospace environment which exploits temporal, frequency and spatial diversity techniques with simple hardware. A custom, static, micro slot, structured beaconing MAC protocol compatible with the industry-popular IEEE802.15.4 PHY and amenable for diversity techniques is used to enhance the robustness of communication. Determinism, programmability as well as scalability are ensured in the design of the MAC layer. The proposed system enables reliable communication and better bandwidth utilization without negative impact on latency or complexity of WSN. Measurements in a highly dynamic flight environment as well as simulations and performance evaluations in terms of packet error rates have shown that the system performs better than state-of-the-art sensor networks under a wide range of boundary conditions, especially in a closed metallic environment.

Even though the design can tolerate a synchronization time slip of $\pm 22 \mu \mathrm{s}$, actual time slip observed is only $\pm 6 \mu$ s under extreme temperature conditions using COTS components. The system is scalable regarding the number of WSNs and the sensors in a single WSN. Any WSN compliant to the beaconing MAC protocol can join the network and communicate without any extra effort. Since vacant time slots are provided in the WBS side, it is not difficult to add a new WSN at a later point of time.

In addition to robust communication, miniaturized packaging of WSNs with RF, in-built MEMS-based sensors and external signal conditioning are demonstrated. Usage of flash-based reprogrammable FPGA for flight applications is also proven and these FPGAs can be used in other applications as well.

This experiment gives an indication that IEEE802.15.4 with a robust MAC is a suitable technology in the 
aerospace environment, although further experimental studies are necessary to assess the reliability and quality of service demands for aerospace applications.

\section{Future work}

Some data losses encountered during flight are being analysed and probable causes along with rectification measures worked out. Design improvements by way of advanced components, board-design and communication protocol are planned in future experiments. Channel equalization and interleaving of the DSSS chip sequence are interesting alternatives to our approach to be investigated. Further work is envisaged to package the WSN in small form factor along with pressure sensors. Techniques such as battery powering, energy harvesting and associated low-power design methodologies are also researched to render the sensors completely tether-free, so that they are amenable to 'plug-and-play' mode of integration. These are expected to contribute largely towards bringing the launch vehicle telemetry and avionics systems in tune with advanced concepts such as integrated modular avionics and reconfigurable architectures $^{14,15}$

1. Pillai, S. S. et al., A versatile, software programmable telemetry system for satellite launch vehicles. In Proceedings of International Telemetering Conference (ITC 06), San Diego, USA, October 2006.

2. Jose, S. et al., Instrumentation and baseband telemetry for RLV-TD Hex mission. J. Inst. Eng. (India): Ser. C, 2017, 98(6), 697-704.

3. Varghese, B., Sreelal, S., Sreekumar, S., Vinod, P. and Mookiah, T., IEEE802.15.4 based efficient beaconing MAC for real-time aerospace applications. In IEEE International Conference on Signal Processing, Informatics, Communication and Energy Systems (IEEE SPICES 2015), NIT Calicut, February 2015.

4. Varghese, B., John, N. E., Sreelal, S. and Gopal, K., Design and development of an RF Energy Harvesting Wireless Sensor Node (EH-WSN) for aerospace applications. In Sixth International
Conference on Advances in Computing and Communications (ICACC 2016), Cochin, September 2016; Reprinted in Procedia Comput. Sci., 2016, 93, 230-237.

5. Kesuma, H., Sebald, J., Ahobala, T. and Paul, S., Ariane 5 Space Launcher Vehicle Equipment Bay Wireless Sensor Network Telemetry Subsystem with Smart Sensors, In 36th European Telemetry and Test Conference, Nuremberg, Germany, 2016, pp. 233-238.

6. Tati, D., Klaue, J. and Sebald, J., Reliable real-time wireless sensor networks using spatial diversity. In IEEE International Conference on Wireless for Space and Extreme Environments (WiSEE), Aachen, Germany, 2016.

7. Blanckenstein, J., Nardin, C., Klaue, J. and Karl, H., Error characterization of multi-access point WSN's in an aircraft cabin. In ICC 2015 Workshop on Dependable Vehicular Communications, June 2015.

8. http://www.hartcomm.org (accessed on October 2018).

9. http://www.isa.org (accessed on October 2018).

10. http://www.industrialwireless.cn (accessed on October 2018).

11. Ferrer Coll, J., Channel characterization and wireless communication performance in industrial environments. Ph.D. thesis, KTH Royal Institute of Technology, Stockholm, Sweden, 2014.

12. Tanghe, E. et al., The industrial indoor channel: large-scale and temporal fading at 900, 2400, and $5200 \mathrm{MHz}$. IEEE Trans. Wireless Commun., 2008, 7(7), 2740-2751.

13. IEEE 802.15.4 Standard: Wireless medium access control (MAC) and physical layer (PHY) specifications for low-rate wireless personal area networks (WPANs). 2006, pp. 1-323.

14. Burke, E., Aerospace vehicle scalable, modular and reconfigurable technologies to bring innovation and affordability. In 30th Space Symposium, Colorado, USA, May 2014.

15. Insaurralde, C., Reconfigurable computer architectures for dynamically adaptable avionics systems. IEEE Aerosp. Electron. Syst. Mag., 2015, 30(9), 46-53.

ACKNOWLEDGEMENTS. We acknowledge the motivation provided by Dr K. Sivan (former Director, VSSC, Thiruvananthapuram and present Chairman, ISRO) and Dr S. Unnikrishnan Nair (Project Director, HSP) for the piggy-back flight test of the WIS payload in HSP-PAT mission. We thank S. Somanath (Director, VSSC) for granting permission and providing guidance to publish this work.

doi: $10.18520 / \mathrm{cs} / \mathrm{v} 120 / \mathrm{i} 1 / 152-160$ 